\title{
Typical Ground States for Large Sets of Interactions
}

\author{
Aernout van Enter $^{1}$ D $\cdot$ Jacek Miękisz ${ }^{2}$
}

Received: 18 July 2020 / Accepted: 23 September 2020 / Published online: 29 September 2020

(c) The Author(s) 2020

\begin{abstract}
We discuss what ground states for generic interactions look like. We note that a recent result, due to Morris, implies that the behaviour of ground-state measures for generic interactions is similar to that of generic measures. In particular, it follows from his observation that they have singular spectrum and that they are weak mixing, but not mixing.
\end{abstract}

Keywords Generic behaviour · Generic interactions · Lattice-gas models · Ground states · Non-periodic order · Quasicrystals

\section{Introduction: Generic Behaviour}

It is an important question of statistical physics what the properties of ground states, Gibbs states, phase diagrams are.

It is common to start this question in the context of classical statistical mechanics, and often the problem is even more simplified by studying the zero-temperature question, the ground state problem. Until around 1980 there was a consensus among condensed-matter physicists that it was to be expected that usually ground states, and in higher dimensions also low-temperature equilibrium states, are crystalline, displaying some kind of periodicity.

One justification for this belief was the fact that the densest packing of hard spheres was known (in $d=1$ or $d=2$, now also in $d=3, d=8$ and $d=24$ [14,25,71]) or conjectured (in various other dimensions) to be periodic. It is possible to rephrase this densest-packing

Dedicated to Joel Lebowitz on the occasion of his 90th birthday. With best wishes and many thanks for being such a stimulus and such an inspiration, as a researcher, as an editor, as a conference organiser, and as a human being. He has been a role model for both of us for as many years as we have been working in science.

Communicated by Christian Maes.

$\triangle \quad$ Aernout van Enter

a.c.d.van.enter@rug.nl

Jacek Miękisz

miekisz@mimuw.edu.pl

1 Bernoulli Institute, Groningen University, Nijenborgh 9, 9747AG Groningen, Netherlands

2 Institute of Applied Mathematics and Mechanics, University of Warsaw, Banacha 2, 02-097 Warsaw, Poland 
question as the study of the behaviour of the ground states for a classical gas with hard-core interactions.

Densest packings of more general and varying shapes, however, have led to the study of tiling problems. It was discovered, by Robinson and later by Penrose, that there exist finite sets of tiles which enforce quasiperiodicity. Again, tiling problems can be rephrased as ground state problems for nearest-neighbour interactions, where different tiles represent different particles, and even before the experimental discovery of quasicrystals, in mathematics the assumption of ubiquitous periodic behaviour was starting to be put in doubt. For an early description of the connection between tiling problems and ground state problems see e.g. $[52,53]$ or [45].

Another research field developed by the study of ground states for particular, physically plausible, models. In various examples, in particular for Lennard-Jones and similar potentials, mostly in $\mathrm{d}=1$, see e.g. [23], but also more recently for $\mathrm{d}=2$. see e.g. [17,67], it was proven that ground states are periodic.

The Crystal Problem has been reviewed in [52], later in [37] and even more recently in [7].

One further simplification we will employ (as has been also discussed to some extent in the above reviews) is that we study lattice models. This considerably simplifies the problem, as any underlying periodic structure, which in continuous models is very hard to show, even though it might occur (whether due to some hard-core or to some Lennard-Jones-like terms in the interaction), now comes for free. On top of that, an aperiodic placing of particles on lattice sites could occur.

Moreover one might argue, especially in lower dimension at positive temperatures, that lattice models may not be that physically realistic. Still, the study of lattice models has proved to be invaluable in the the understanding of various forms of long-range order occurring in a wide variety of physical systems. And the more serious objections to using lattice models, even at $T=0$, do not apply with the same force to understanding what happens in longerrange models, nor do they really apply to attempts to understand what may occur in the physical dimension $d=3$.

One question this issue of various types of long-range order has led to, and which we will discuss here, is about the behaviour of equilibrium or ground states for typical interactions. An old result due to Gallavotti and Miracle [21], using a theorem of Mazur [38], implies that the set of interactions having a unique tangent to the pressure (= equilibrium state), is generic (that is, it is a dense $G_{\delta}$, a countable intersection of dense open sets). This result holds in any of the commonly studied (Banach) spaces of lattice interactions. The largest interaction space usually considered, $B_{0}$, can be associated to the space of continuous functions, modulo translations, see e.g. [28]. In this space, a number of "pathological" properties can be proven, e.g. there is a dense set of interactions having uncountably many ergodic equilibrium or ground states, $[28,30,62,63]$, the pressure is never Fréchet differentiable [16], the pressure can depend on boundary conditions, and "Ideally Metastable" states [60] as well as "frozen" low-temperature states $[11,12,18]$ can exist.

Later results were proven on the triviality of generic phase diagrams (implying the generic violation of the Gibbs Phase Rule) for various spaces of long-range interactions [29,32] .

More recently, the study of ground states in the guise of "ergodic optimisation" $[8,35]$ has reinvigorated the interest in such questions. For the space of continuous functions, it was proven that ground states for generic interactions have full support, and zero entropy $[9,10,72]$. 
Even more recently, Morris [47] proved a result that properties of generic measures are inherited by ground state measures of generic continuous functions. He applied this to show that they are not mixing.

Here we add the observation in our Corollary 2 that they are weak mixing and have singular (Dynamical and thus Diffraction) spectrum. This follows from the known equivalent statements for generic measures, due to Halmos [26], Rohlin[55], and Knill [36].

Another, maybe not too surprising, consequence, using another result of Israel [31], is that ground states for typical (generic) interactions cannot be Gibbs states (for possibly different interactions).

We remark, by the way, that Simon's "Wonderland" theorem has before provided a number of other examples of problems where singular spectrum of some kind turns out to be generic [65].

Moreover we discuss and speculate on properties of ground states and equilibrium states (which then can be Gibbs states in the DLR sense) in smaller interaction spaces.

One of the fundamental problems in statistical physics is to understand why matter at low temperatures and high enough pressures possesses some sort of long-range order. For ages this was interpreted to mean that matter is crystalline, that is, its constituents, atoms or molecules, form some kind of a three-dimensional lattice. The famous, and still not solved, Crystal Problem is to show that for "reasonable" physical interactions between particles, the arrangements minimizing their energy density are attained by periodic configurations. Periodicity is the strongest embodiment of a positional long-range order.

One major physical reason which has spurred the interest in the behaviour of "typical" ground states was the discovery of quasicrystals [61]. Despite earlier beliefs and claims that all or most physical systems should have crystalline, periodically ordered, ground states and low-temperature states, such claims have turned out to be dubious and in many contexts untrue. For an early rigorous result contradicting this crystalline paradigm, see for example [46]. Especially since Shechtman's discovery of the first quasicrystals [61], which led to his 2011 Nobel Prize in Chemistry, there has developed a large amount of research, experimental, theoretical and also mathematical, about the properties of quasicrystals, the nature of the associated aperiodic order and related questions. See for example the books [5,59].

For a recent, more physics-style paper, again showing that periodic order is generically not to be expected, see for example [20].

The discovery of quasicrystals showed us that other forms of long-range order than periodic ones might be present in Nature. The non-periodic order of quasicrystals was represented by a (dense) discrete spectrum in X-ray experiments. From a different point of view, energyminimizing (or free-energy-minimizing) configurations of particles gave rise to non-mixing ground-state (or Gibbs state) measures. Many (toy) examples were constructed with such properties, some of them based on previously constructed non-periodic tilings [39-42,49] The natural question then arises: how typical (generic) and how robust are such examples? It was proven in $[44,46,50]$ that in the Banach space of two-body summable interactions, for typical interactions, that is in a dense $G_{\delta}$ set, the ground-state measure is non-periodic, non-mixing and has a zero entropy. In [41], a classical lattice-gas model was constructed with a non-periodic ground-state measure which is stable against small perturbations of nearest-neighbour interactions.

In this note we review and combine some old results on generic properties of latticegas models with recent ideas from ergodic optimization, and present the new result that for generic interactions a ground state is non-mixing, but weakly mixing, and moreover it has a singular (dynamical and thus diffraction) spectrum. It means that generically ground states are quite disordered but will still have some long-range order. 
Our result is based on the recent result of Morris [47] on ergodic optimization for generic continuous functions. In particular he showed that ergodic measures which maximize the integral of a generic continuous function have the same properties as generic ergodic measures.

In Sect. 2, we introduce classical lattice-gas models. We also review some old results concerning generic presence of quasi-crystalline or weak crystalline ground states.

In Sect. 3, we show how our result follows from that of Morris.

In Sect. 4, we discuss some open problems and directions of future research.

\section{Classical Lattice-Gas Models}

We will consider the case of classical, finite-spin lattice systems, as for example discussed in $[19,24,28,56,64,68]$. Our formulation mainly follows Israel [28].

Our configuration space is $\Omega=\Omega_{0} Z^{d}$, with $\Omega_{0}$ finite. Translations on $Z^{d}$ are indicated by $\tau_{x}, x \in Z^{d}$.

Translation-invariant interactions $\Phi$ are sets of functions $\Phi_{X}$ on $\Omega_{0}{ }^{X}$, with $X$ a finite subset of $Z^{d}$, and such that the $\Phi_{X}$ are translation-invariant.

Different interaction (Banach) spaces can be defined by different norms, using different translation-invariant functions $f$ on the subsets of $Z^{d}$, by $\left\|\mid \Phi_{X}\right\|\left\|_{f}=\sum_{0 \in X}\right\| \Phi_{X} \| f(X)$.

Often the $f$ one chooses depends on either $|X|$, the cardinality of $X$, or on its diameter $\operatorname{diam}(X)$. The largest interaction space we will consider is $B_{0}$, which is obtained by choosing $f(X)=\frac{1}{X}$. Other commonly used interaction spaces are $B_{n}$, defined by taking $f(X)=$ $|X|^{n-1}$, and $B_{\lambda, \text { exp }}$, defined by $f(X)=e^{\lambda|X|}$.

If it is the case that $\Phi_{X}=0$ for all $X$ with large enough diameter, we say that our interaction is of finite range; if it is the case that $\Phi_{X}=0$ when $X$ contains more than two sites, we say that we are considering pair interactions.

To each interaction $\Phi$ is associated a continuous function on $\Omega$, describing the energy per site, localised around the origin, $A_{\Phi}=\sum_{0 \in X} \frac{\Phi_{X}}{|X|}$. Translation-invariant measures on $\Omega$ correspond to bounded linear functionals on $B_{0}$, in an isometric way (see e.g. [28], Lemma II.1.1).

On $B_{0}$ one can define a Lipschitz continuous and convex pressure function $P$. On the set of translation-invariant probability measures on $\Omega$ one can define an affine entropy (density) function $s$.

Pressure and entropy are each other's Legendre-Fenchel transforms, and are related by dual variational principles:

$$
P(\Phi)=\sup \left(s(\mu)-\mu\left(A_{\Phi}\right) \mid \mu \in E_{I}\right)
$$

and

$$
s(\mu)=\inf \left(P(\Phi)+\mu\left(A_{\Phi} \mid \Phi \in B_{0}\right)\right.
$$

Solutions of these variational principles satisfy

$$
P(\Phi)=s\left(\mu_{\Phi}\right)-\mu_{\Phi}\left(A_{\Phi}\right) .
$$

In the case that a measure $\mu_{\Phi}$ solves the variational principle, we say that $\mu_{\Phi}$ is an equilibrium state for $\Phi$. Such an equilibrium state corresponds to a tangent functional to the pressure function, tangent to $\mathrm{P}$ at the point $\Phi$.

If we replace the affine entropy function $s(\mu)$ by 0 , the corresponding measure becomes a ground state measure. 
Remark In ergodic optimization, see e.g. [8,35], usually one considers maximizing, rather than minimizing (ground state) measures, but the questions are easily seen to be equivalent by a simple sign change.

The fact that convex functions on Banach spaces generically have a unique tangent implies that generically there exists a unique translation invariant equilibrium or ground state $[21,57]$.

\section{Generic Measures and Generic Interactions, Corollaries of a Theorem by Morris}

In [47] the following Theorem was proven. In our setting it says the following:

\section{Morris' Theorem}

Let $U$ be a generic set of translation-invariant measures on $\Omega$. Then the set of functions $V$ whose ground states are in $U$ is generic (a $G_{\delta}$ in the space of continuous functions on $\Omega$ ).

In words, it says that if a generic set of measures has a certain property, this same property holds for the ground states of a generic set of interactions in $C(\Omega)\left(\right.$ orin $\left.B_{0}\right)$.

Corollary 1 (Morris): In particular, it was concluded by Morris that generic ground states (= maximizing measures) are unique, non-mixing, have full support, and have entropy zero.

Corollary 2 Ground states for generic interactions in $C(\Omega)$ are weak mixing and have singular diffraction spectrum. Moreover they cannot be written as Gibbs measures for any interaction.

Proof This follows directly from the fact that those properties are known to hold for generic, translation-invariant measures. These results are due respectively to Halmos [26], Knill [36], and Israel [31].

We notice that in $C\left(\Omega_{0}\right)$ there are dense sets of interactions with uncountably many ergodic ground states $[30,62]$. Thus one cannot expect much more regular behaviour. We do remark, though, that the absence of point spectrum in the pure singular spectrum result shows that generic ground states are neither periodic, nor quasiperiodic. Thus generically neither crystals, not quasicrystals exist (but "weak" [70] or "turbulent" [58] crystals do). Thus is compatible with the results of [44,46,50,51], showing some statistical homogeneity, but excluding periodicity, for generic interactions in various interaction spaces.

\section{Speculations on Different Interaction Spaces}

The space $B_{0}$, although mathematically natural, as one can associate it to the continuous functions, has a number of pathological properties. There exist dense sets (although not generic ones) for which there are uncountably many ergodic (extremal translation-invariant) equilibrium or ground states $[30,62]$, -as is proven via the Bishop-Phelps theorem- generic ground states have full support [9], the pressure can depend on boundary conditions, strict convexity of the pressure does not hold, see e.g. the discussion in [68, Sect. 2.6.7].

This is a reason why often smaller interaction spaces are considered. In the space $B_{1}$, Gibbs measures can be defined, according to the prescriptions of Dobrushin, Lanford and Ruelle, in $B_{2}$, the uniqueness theorem of Dobrushin implies that there are open high-temperature, high-magnetic-field or low-density regimes. In $B_{\lambda, \exp }$ it can be shown that pressure and states can be analytic in open high-temperature or low-density regions, etc. 
Also one can have open sets in those smaller spaces where there are pure, homogeneous ground state configurations (vacua). Typical statements about ground states then distinguish between two cases, the case where there is a unique homogeneous ground state configuration and the case where there is some weak, non-periodic form of long-range order. We suspect that the lack of point spectrum, that is the lack of "crystalline" long-range order, may also hold there.

We remark that Israel [29] has proved that phase coexistence is exceptional, in these interaction spaces. His arguments are written down for positive temperature, but also apply for ground states. It seems that his arguments with minor modifications can be used to obtain a genericity statement excluding periodicity in ground states, in a somewhat more abstract and general context, than the result excluding periodicity for generic interactions as was obtained for lattice-gas models in [46]. In other words, under a generic long-range perturbation of an interaction a unique pure phase (or vacuum) can be stable, but neither phase coexistence, nor periodicity are stable under such perturbations.

One question we don't know how to answer, however, is what happens with aperiodic order under long-range perturbations. Quasicrystalline long-range order is defined in terms of discrete diffraction spectra, whereas weaker forms of long-range order occur when singular spectra appear (weak or turbulent crystals $[58,70]$ ), or even can occur without any spectral indications [66]. As the number of possible periods is countable, excluding all of them leads to considering a countable intersection of dense $G_{\delta}$ sets, which preserves genericity; the number of possible quasicrystalline spectra is uncountable, so a different argument would be required to investigate stability questions of quasicrystalline order.

On the other hand, if we restrict ourselves even more and look at fast decaying interactions in one dimension, corresponding for example with Hölder or Lipschitz functions, sometimes it can be proven that typical ground states behave quite differently, and that periodic ground states are generic $[15,27,48]$.

We note that it has been known for quite some time that any finite-range interaction in one dimension always has periodic ground states $[13,43,54]$. But this is no longer true if one allows even fast decaying interactions in one dimension, or nearest-neighbour interactions based on tilings in higher dimensions, see e.g. [1,22,52,53,69]. For example, it is not known if it is the case that the quasicrystalline order such as occurs in Devil's Staircases [2-4,6,33,34], or in more general Sturmian (balanced) ground states [69], is stable under some class of short-range perturbations.

In higher dimensions for short-range interactions, however, in many cases long-range order is stable, as can be shown by Pirogov-Sinai theory. However, it is not known if genericity statements in the sense of statements holding true for "generic short-range interactions", and predicting that they behave in a certain way as regards their long-range order, are valid.

Acknowledgements We would like to thank the National Science Centre (Poland) for a financial support under Grant No. 2016/22/M/ST1/00536, which made a visit of AvE to Warsaw possible, during which we started this project.

Open Access This article is licensed under a Creative Commons Attribution 4.0 International License, which permits use, sharing, adaptation, distribution and reproduction in any medium or format, as long as you give appropriate credit to the original author(s) and the source, provide a link to the Creative Commons licence, and indicate if changes were made. The images or other third party material in this article are included in the article's Creative Commons licence, unless indicated otherwise in a credit line to the material. If material is not included in the article's Creative Commons licence and your intended use is not permitted by statutory regulation or exceeds the permitted use, you will need to obtain permission directly from the copyright holder. To view a copy of this licence, visit http://creativecommons.org/licenses/by/4.0/. 


\section{References}

1. Aubry, S.: Weakly periodic structures and example. Phys. Colloques 50(C3), 97-106 (1989)

2. Aubry, S.: Complete devil's staircase in the one-dimensional lattice gas. J. Phys. Lett. 44, L247-L250 (1983)

3. Aubry, S.: Exact models with a complete Devil's staircase. Phys. C 16, 2497-2508 (1983)

4. Aubry, S.: Devil's staircase and order without periodicity in classical condensed matter. J. Phys. 44, 147-162 (1983)

5. Bake, M., Grimm, U.: Aperiodic Order. A Mathematical Invitation, vol. 1. Cambridge University Press, Cambridge (2013)

6. Bak, P., Bruinsma, R.: One-dimensional Ising model and the complete Devil's staircase. Phys. Rev. Lett. 49, 151249 (1982)

7. Blanc, X., Lewin, M.: The crystallizaton conjecture: a review. EMS Surv. Math. Sci. 2, 255-306 (2015)

8. Bochi, J.: Ergodic optimization of Birkhoff averages and Lyapunov exponents. Proc. ICM 2018, 18251844 (2019)

9. Bousch, T., Jenkinson, O.: Cohomology classes of dynamically non-negative $C^{k}$ functions. Invent. Mat. 148, 207-217 (2002)

10. Brémont, J.: Entropy and maximizing measures of generic continuous functions. Compt. R. Acad. Sci. Ser. I 346, 199-201 (2008)

11. Bruin, H., Leplaideur, R.: Renormalization, thermodynamic formalism and quasi-crystals in subshifts. Commun. Math. Phys. 321, 209-247 (2013)

12. Bruin, H., Leplaideur, R.: Renormalization, freezing phase transition, and Fibonacci quasicrystals. Ann. Sci. l'ENS 48, 739-763 (2015)

13. Bundaru, M., Angelescu, N., Nenciu, G.: On the ground state of Ising chains with finite range interactions. Phys. Lett. 43A, 5-6 (1973)

14. Cohn, H., Kumar, A., Miller, S.D., Radchenko, D., Viazovska, M.: The sphere packing problem in dimension 24. Annals of Mathematics. 185(3), 1017-1033 (2017)

15. Contreras, G.: Ground states are generically a periodic orbit. Invent. Math. 205, 383-412 (2016)

16. Daniëls, H.A.M., van Enter, A.C.D.: Differentiability properties of the pressure in lattice systems. Commun. Math. Phys. 71, 65-76 (1980)

17. De Luca, L., Friesecke, G.: Crystallization in two dimensions and a discrete Gauss-Bonnet theorem. J. Nonlinear Sci. 28, 69-90 (2018)

18. Fisher, M.E.: On discontinuity of the pressure. Commun. Math. Phys. 26, 6-14 (1972)

19. Friedli, S., Velenik, Y.: Statistical mechanics of lattice systems: a concrete mathematical introduction. Cambridge University Press, Cambridge (2018)

20. Friedrich, M., Stefanelli, U.: Crystallization in a one-dimensional periodic landscape. J. Stat. Phys. 179, 485-501 (2020)

21. Gallavotti, G., Miracle-Sole, S.: Statistical mechanics of lattice systems. Commun. Math. Phys. 5, 317323 (1967)

22. Gardner, C.S., Miękisz, J., Radin, C., van Enter, A.C.D.: Fractal symmetry in an Ising model. J. Phys. A: Math. Gen. 22, L1019-L1023 (1989)

23. Gardner, C.S., Radin, C.: The infinite-volume ground state of the Lennard-Jones potential. J. Stat. Phys. 20, 719-724 (1979)

24. Georgii, H.-O.: Gibbs Measures and Phase Transitions, 2nd edn. De Gruyter, Berlin (2011)

25. Hales, T.C.: A proof of the Kepler conjecture. Ann. Math. Second Ser. 162(3), 1065-1185 (2005)

26. Halmos, P.R.: In general a measure preserving transformation is mixing. Ann. Math. 45, 786-792 (1944)

27. Huang, W., Lian, Z., Ma, X., Xu, L., Zhang, Y.: Ergodic optimization theory for a class of typical maps. arXiv:1904.01915v2 (2019)

28. Israel, R.B.: Convexity in the Theory of Lattice Gases. Princeton University Press, Princeton (1979)

29. Israel, R.B.: Generic triviality of phase diagrams in spaces of long-range interactions. Commun. Math. Phys. 106, 459-466 (1986)

30. Israel, R.B.: Existence of phase transitions in spaces of long-range interactions. Commun. Math. Phys. 43, 59-68 (1975)

31. Israel, R.B.: Some generic results in mathematical physics. Markov Proc. Rel. Fields 10, 517-521 (2004)

32. Israel, R.B., Phelps, R.R.: Some convexity questions arising in statistical mechanics. Math. Scand. 54, 133-156 (1984)

33. Jędrzejewski, J., Miękisz, J.: Devil's staircase for non-convex interactions. Europhys. Lett. 50, 307-311 (2000)

34. Jȩdrzejewski, J., Miękisz, J.: Ground states of lattice gases with "almost" convex repulsive interactions. J. Stat. Phys. 98, 589-620 (2000) 
35. Jenkinson, O.: Ergodic optimization. Discret. Cont. Dyn. Syst. 15, 197-224 (2006)

36. Knill, O.: Singular continuous spectrum and quantitative rates of weak mixing. Discret. Cont. Dyn. Syst. 4, 33-42 (1998)

37. Le Bris, C., Lions, P.-L.: From atoms to crystals: a mathematical journey. Bull. Am. Math. Soc. 42(3), 291-363 (2005)

38. Mazur, S.: Über Konvexen Mengen in linearen normierten Räumen. Studia Math. 4, 70-84 (1933)

39. Miękisz, J.: Many phases in systems without periodic ground states. Commun. Math. Phys. 107, 577-586 (1986)

40. Miękisz, J.: A microscopic model with quasicrystalline properties. J. Stat. Phys. 58, 1137-1149 (1990)

41. Miękisz, J.: Stable quasicrystalline ground states. J. Stat. Phys. 88, 691-711 (1997)

42. Miękisz, J.: Classical lattice-gas models of quasicrystals. J. Stat. Phys. 97, 835-850 (1999)

43. Miękisz, J., Radin, C.: The Third Law of thermodynamics. Mod. Phys. Lett. B 1, 61-65 (1987)

44. Miękisz, J.: How low temperature causes long-range order. J. Phys. A 21, 529-531 (1988)

45. Miękisz, J.: Quasicrystals-Microscopic Models of Nonperiodic Structures. Leuven Notes on Mathematical and Theoretical Physics. Leuven University Press, Leuven (1993)

46. Miękisz, J., Radin, C.: Why solids are not really crystalline. Phys. Rev. B 39, 1950-1952 (1989)

47. Morris, I.D.: Ergodic optimization for generic continuous functions. Discret. Cont. Dyn. Syst. 27, 383-388 (2010)

48. Morro, M., Sant'Anna, R., Varandas, P.: Ergodic optimization for hyperbolic flows and Lorenz attractors. Ann. Henri Poincaré 21, 3253-3283 (2020)

49. Radin, C.: Crystals and quasicrystals: a lattice gas model. Phys. Lett. 114A, 381-383 (1986)

50. Radin, C.: Ordering in lattice gases at low temperature. J. Phys. A 22, 317-319 (1989)

51. Radin, C.: Correlations in classical ground states. J. Stat. Phys. 43, 707-712 (1986)

52. Radin, C.: Low temperature and the origin of crystalline symmetry. Int. J. Mod. Phys. B 1, 1157-1191 (1987)

53. Radin, C.: Global order from local sources. Bull. Am. Math. Soc. 25, 335-364 (1991)

54. Radin, C., Schulman, L.S.: Periodicity of classical ground states. Phys. Rev. Lett. 51, 1621-1622 (1983)

55. Rokhlin, V.A.: A general measure-preserving transformation is not mixing. (Russian). Dokl. Akad. Nauk SSSR 60, 349-351 (1948)

56. Ruelle, D.: Thermodynamic Formalism. Cambridge Mathematical Library, 2nd edn. Cambridge University Press, Cambridge (2004)

57. Ruelle, D.: Some remarks on the ground state of infinite systems in statistical mechanics. Commun. Math. Phys. 11, 339-345 (1969)

58. Ruelle, D.: Do turbulent crystals exist? Phys. A 113, 619-623 (1982)

59. Senechal, M.: Quasicrystals and Geometry. Cambridge University Press, Cambridge (1995)

60. Sewell, G.: Stability, equilibrium and metastability in statistical mechanics. Phys. Rep. 57, 307-342 (1980)

61. Shechtman, D., Blech, I., Gratias, D., Cahn, J.W.: Metallic phase with long-range orientational order and no translational symmetry. Phys. Rev. Lett. 53, 1951 (1984)

62. Shinoda, M.: Uncountably many maximizing measures for a dense subset of continuous functions. Nonlinearity 31, 2192-2200 (2018)

63. Shinoda, M., Takahasi, H.: Lyapunov optimization for non-generic one-dimensional expanding Markov maps. Ergodic Th. Dyn. Syst. 40(9), 2571-2592 (2020)

64. Simon, B.: The Statistical Mechanics of Lattice Gases. Princeton University Press, Princeton (1993)

65. Simon, B.: Operators with singular continuous spectrum: I. General operators. Ann. Math. 141, 131-145 (1995)

66. Slawny, J.: Ergodic properties of equilibrium states. Commun. Math. Phys. 80, 477-483 (1981)

67. Theil, F.: A proof of crystallization in two dimensions. Commun. Math. Phys. 262, 209-236 (2006)

68. van Enter, A.C.D., Fernández, R., Sokal, A.D.: Regularity properties and pathologies of position-space renomalization group transformations: scope and limitations of Gibbsian theory. J. Stat. Phys. 72, 8791167 (1993)

69. van Enter, A.C.D., Koivusalo, H., Miękisz, J.: Sturmian ground states in classical lattice-gas models. J. Stat. Phys. 178, 832-844 (2020)

70. van Enter, A.C.D., Miękisz, J.: How should one define a (weak) crystal? J. Stat. Phys. 66, 1147-1153 (1992)

71. Viazovska, M.: The sphere packing problem in dimension 8. Ann. Math. 185(3), 991-1015 (2017) 
72. Yang, D., Zhang, J.: Ergodic optimization for some dynamical systems beyond uniform hyperbolicity. arXiv:2005.09315v2 (2020)

Publisher's Note Springer Nature remains neutral with regard to jurisdictional claims in published maps and institutional affiliations. 